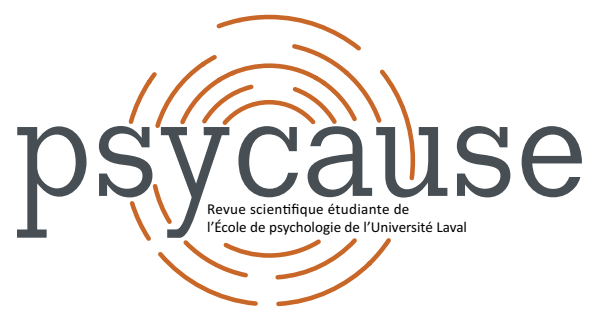

\title{
PSYCAUSE
}

Revue scientifique étudiante de l'École de psychologie de l'Université Laval

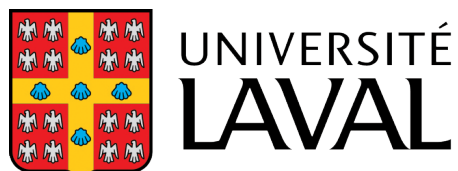

Faculté des sciences sociales École de psychologie

NOVEMBRE 2020 - VOL. $10 \mathrm{~N}^{\circ} 2$

\section{EFFETS DE L'UTILISATION D'INSTAGRAM SUR L'INSATISFACTION CORPORELLE DES JEUNES FEMMES}

Amélie CHARTRAND ${ }^{1}$, Émélie LAVOIE ${ }^{1}$, Aurélie LEMAY-LANGLOIS ${ }^{1} \&$ Marie-Claude RICHARD ${ }^{1}$

${ }^{1}$ École de psychologie, Université Laval

*amelie.chartrand.2@ulaval.ca

\section{Pour citer l'article}

Chartrand, A., Lavoie, É., Lemay-Langlois, A., \& Richard, M.-C. (2020). Effets de I'utilisation d'Instagram sur l'insatisfaction corporelle des jeunes femmes. Psycause: Revue scientifique étudiante de l'École de psychologie de l'Université Laval, 10(2), 5-6. 


\title{
EFFETS DE L'UTILISATION D'INSTAGRAM SUR L'INSATISFACTION CORPORELLE DES JEUNES FEMMES
}

\author{
Amélie CHARTRAND ${ }^{1}$, Émélie LAVOIE ${ }^{1}$, Aurélie LEMAY-LANGLOIS ${ }^{1}$ \& Marie-Claude RICHARD ${ }^{1}$ \\ ${ }^{1}$ École de psychologie, Université Laval \\ *amelie.chartrand.2@ulaval.ca
}

\section{Mots-clés : Instagram, insatisfaction corporelle, littératie médiatique, transition à la vie adulte}

Le passage de l'adolescence à l'âge adulte est une période critique pour le développement de l'identité durant laquelle I'insatisfaction corporelle constitue un enjeu important (Bucchianeri, Arikian, Hannan, Eisenberg \& NeumarkSztainer, 2013). Celle-ci entraîne notamment une réduction de la qualité de vie reliée à la santé physique et mentale (Griffiths et coll., 2016). L'utilisation d'Instagram (IG) est susceptible de renforcer ce risque en raison de son contenu exclusivement visuel, particulièrement chez les jeunes femmes (Wiederhold, 2019). En favorisant une internalisation d'un idéal de minceur par la comparaison sociale, une faible estime de soi et une insatisfaction corporelle (Sherlock \& Wagstaff, 2018), I'utilisation d'IG affecterait la santé mentale des femmes de 18 à 24 ans formant la cohorte démographique la plus active sur IG au Québec (CEFRIO, 2018). II existe néanmoins un consensus concernant l'implication de la littératie médiatique, soit l'autonomie critique envers les médias en général, dans la relation entre leur utilisation et leurs effets négatifs (McLean, Paxton \& Wertheim, 2016). Une étude suggère plus précisément que la fréquence d'utilisation d'IG est liée à l'insatisfaction corporelle et que la littératie médiatique modère cette relation (Melioli et coll., 2018).

La présente étude vise à mieux comprendre les enjeux identitaires liés à l'image corporelle des jeunes femmes en transition à la vie adulte. Considérant qu'il y a une absence d'outils valides et standards pour mesurer l'utilisation d'IG,

\section{Tableau 1}

Corrélations de Spearman entre les variables à l'étude et leurs statistiques descriptives

\begin{tabular}{|c|c|c|c|c|c|c|c|c|}
\hline & 1 & 2 & 3 & 4 & 5 & 6 & 7 & 8 \\
\hline 1. Ratio photos de soi sur photos totales & & $0,648^{\star \star}$ & $0,897^{\star *}$ & 0,172 & 0,132 & 0,069 & 0,019 & 0,054 \\
\hline 2. Photos publiées & & & $0,819^{\star \star}$ & 0,058 & 0,168 & 0,211 & $-0,241^{*}$ & 0,007 \\
\hline 3. Photos de soi publiées & & & & 0,138 & 0,126 & 0,153 & $-0,140$ & $-0,084$ \\
\hline 4. Abonnements & & & & & 0,657 & $0,365^{\star \star}$ & 0,020 & $-0,201$ \\
\hline 5. Abonné.e.s & & & & & & $0,380 * \star$ & $-0,163$ & $-0,160$ \\
\hline 6. Fréquence quotidienne d'utilisation & & & & & & & $-0,234^{*}$ & $-0,254^{*}$ \\
\hline 7. Insatisfaction corporelle & & & & & & & & $-0,005$ \\
\hline \multicolumn{9}{|l|}{ 8. Littératie médiatique } \\
\hline$M$ & 0,424 & 1,390 & 1,026 & 451,649 & 480,948 & 39,857 & 42,942 & 57,046 \\
\hline ET & 0,466 & 2,363 & 1,999 & 451,649 & 418,969 & 52,042 & 14,632 & 12,791 \\
\hline
\end{tabular}

Note. $M=$ moyenne; $E T=$ écart-type ; ${ }^{*} p<0,05 ;{ }^{* \star} p<0,001$ le premier objectif est de vérifier si le ratio du nombre de photos de soi publiées par rapport au nombre total de photos publiées dans le dernier mois (ratio) en est une mesure représentative. Celle-ci est priorisée considérant la place prépondérante de l'apparence sur IG. Le deuxième objectif est d'examiner la relation entre l'utilisation d'IG, en hausse dans la population générale (CEFRIO, 2018), etl'insatisfaction corporelle. Le troisième objectif est d'explorer le rôle médiateur de la littératie médiatique dans cette relation, car son rôle protecteur envers la satisfaction corporelle lors de I'utilisation d'autres médias pourrait s'appliquer à IG.

\section{Méthode}

Un total de 77 participantes compose l'échantillon à l'étude. Les critères d'inclusion sont de s'identifier au genre féminin ou non-binaire, d'être âgées de 18 à 24 ans et de maîtriser la langue française. Les participantes répondent à un questionnaire réalisé sur LimeSurvey et accessible sur les appareils électroniques via un hyperlien diffusé sur Facebook. Le questionnaire, d'une durée de 20 minutes, est composé de quatre sections. La première est constituée d'un questionnaire sociodémographique maison. La deuxième évalue, via des questions maison, les différentes mesures liées à l'utilisation d'IG (Tableau 1). La troisième correspond à l'Échelle d'Estime Corporelle (Mendelson \& White, 1982), un instrument d'autoévaluation mesurant l'insatisfaction corporelle. Finalement, 
une version de 10 items provenant des 20 items originaux du Media Attitudes Questionnaire (MAQ; Irving, Dupen \& Berel, 1998) est utilisée afin de mesurer la littératie médiatique.

\section{Résultats et discussion}

Pour ce qui est du premier objectif 1 , le ratio s'avère non corrélé aux autres mesures d'utilisation d'IG et à I'insatisfaction corporelle, permettant ainsi de constater qu'il n'est pas une mesure représentative de I'utilisation d'IG. La fréquence quotidienne d'utilisation d'IG étant la variable qui corrèle significativement et positivement avec le plus de variables à l'étude, c'est cette mesure qui sera utilisée pour l'ensemble des analyses subséquentes (Tableau 1).

Quant au deuxième objectif, les données indiquent que la fréquence d'utilisation d'IG est négativement et significativement corrélée à l'insatisfaction corporelle. Ainsi, plus les femmes passent de temps sur IG, moins elles ressentent de I'insatisfaction corporelle. Cela pourrait s'expliquer par le type de contenu visionné par l'échantillon, peu lié à l'image corporelle. Par ailleurs, parmi les participantes consommant ce type de contenu, la majorité s'expose au mouvement de body positivity, faisant la promotion de l'acceptation corporelle. Finalement, les jeunes femmes ayant une insatisfaction corporelle élevée pourraient éviter de s'exposer à des modèles inatteignables de beauté par le biais d'IG afin d'en diminuer les impacts (Rousseau, Eggermont \& Frison, 2017).

Étant donné que les postulats de base de l'analyse de médiation ne sont pas respectés, le rôle modérateur de la littératie médiatique a été vérifié. Ce dernier s'est avéré non significatif, indiquant que la littératie médiatique ne joue un rôle ni médiateur ni modérateur dans la relation d'intérêt. Puisque cette relation n'a jamais été vérifiée pour la population des jeunes femmes québécoises, il se peut que le contexte culturel explique ce résultat. En apportant quelques modifications pertinentes à l'étude en lien avec le nombre de participantes ou le questionnaire utilisé, les résultats pourraient différer.

Cette recherche permet de pallier certaines lacunes identifiées dans les écrits consultés. Elle éclaire notamment le caractère multifactoriel de la relation entre l'utilisation d'IG et l'insatisfaction corporelle. Elle fournit également des pistes d'intervention et des sujets d'études futures autour des notions de body positivity et de littératie médiatique.

\section{Pour citer l'article}

Chartrand, A., Lavoie, É., Lemay-Langlois, A., \& Richard, M.-C. (2020). Effets de l'utilisation d'Instagram sur l'insatisfaction corporelle des jeunes femmes. Psycause: Revue scientifique étudiante de l'École de psychologie de l'Université Laval, 10(2), 5-6.

\section{Références}

Bucchianeri, M. M., Arikian, A. J., Hannan, P. J., Eisenberg, M. E., \& Neumark-Sztainer, D. (2013). Body dissatisfaction from adolescence to young adulthood: Findings from a 10-year longitudinal study. Body Image, 10(1), 1-7. http:// doi.org/10.1016/j.bodyim.2012.09.001

CEFRIO. (2018). L'usage des médias sociaux au Québec. NETendances, 9(5). Repéré à https://transformation-numerique.ulaval.ca/wp-content/uploads/2020/09/netendances-2018-usage-medias-sociaux.pdf

Griffiths, S., Hay, P., Mitchison, D., Mond, J. M., McLean, S. A., Rodgers, B., \& Paxton, S. J. (2016). Sex differences in the relationships between body dissatisfaction, quality of life and psychological distress. Australian and New Zealand Journal of Public Health, 40(6), 518-522. http://doi. org/10.1111/1753-6405.12538

Irving, L. M., Dupen, J., \& Berel, S. (1998). A media literacy program for high school females. Eating Disorders, 6(2), 119-131. http://doi.org/10.1080/10640269808251248

McLean, S. A., Paxton, S. J., \& Wertheim, E. H. (2016). Does media literacy mitigate risk for reduced body satisfaction following exposure to thin-ideal media? Journal of Youth and Adolescence, 45(8), 1678 1695. http://doi. org/10.1007/s10964-016-0440-3

Melioli, T., Gonzalez, N., El Jazouli, Y., Valla, A., Girard, M., Chabrol, H., \& Rodgers, R. F. (2018). Utilisation d'Instagram, aptitude à critiquer les médias et symptômes de troubles du comportement alimentaire chez les adolescentes: Une étude exploratoire. Journal de Thérapie Comportementale et Cognitive, 28(4), 196 203. http://do.org/10.1016/j. jtcc.2018.06.003

Mendelson, B. K., \& White, D. R. (1982). Relation between Body-Esteem and Self-Esteem of Obese and Normal Children. Perceptual and Motor Skills, 54(3), 899- 905. http://doi.org/10.2466/pms.1982.54.3.899

Rousseau, A., Eggermont, S., \& Frison, E. (2017). The reciprocal and indirect relationships between passive Facebook use, comparison on Facebook, and adolescents' body dissatisfaction. Computers in Human Behavior, 73, 336-344. http://doi.org/10.1016/j.chb.2017.03.056

Sherlock, M., \& Wagstaff, D. L. (2018). Exploring the relationship between frequency of Instagram use, exposure to idealized images, and psychological well-being in women. Psychology of Popular Media Culture, 8(4), 482 490. http://doi. org/10.1037/ppm0000182

Wiederhold, B. K. (2019). Instagram: Becoming a worldwide problem? Cyberpsychology, Behavior, and Social Networking, 22(9), 567 568. http://doi.org/10.1089/cyber.2019.29160.bkw 